\title{
Differentiation of mesenchymal stem cells into cardiomyocytes is regulated by miRNA-1-2 via WNT signaling pathway
}

\author{
Xing Shen ${ }^{1,2}$, Bo Pan', Huiming Zhou', Lingjuan Liu', Tiewei Lv', Jing Zhu', Xupei Huang ${ }^{3}$ and Jie Tian ${ }^{1 *}$
}

\begin{abstract}
Background: Bone marrow derived stem cells (BMSCs) have the potential to differentiate into cardiomyocytes, but the rate of differentiation is low and the mechanism of differentiation is unclear completely. Here, we aimed to investigate the role of miR1-2 in differentiation of mouse BMSCs into cardiomyocyte-like cells and reveal the involved signaling pathways in the procedure.

Methods: Mouse BMSCs were treated with miR1-2 and 5-azacytine (5-aza). The expression of cardiac cell markers: NKx2.5, cTnl and GATA4 in BMSCs were examined by qPCR. The apoptosis rate was detected by flow cytometry and the activity of the $\mathrm{Wnt} / \beta$-catenin signaling pathway was evaluated by measuring the upstream protein of this signaling pathway.

Results: After over-expression of miR1-2 in mouse BMSCs, the apoptosis rate was significantly lower than the 5-aza group, while the expressions of cardiac-specific genes: such as Nkx2.5, cTnl and GATA4 were significantly increased compared to the control group and the 5-aza group. Meanwhile, over-expression of miR1-2 in mouse BMSCs enhanced the expression of wnt11, JNK, $\beta$-catenin and TCF in the Wnt/ $\beta$-catenin signaling pathway. Use of LGK-974, an inhibitor of Wnt/ $\beta$-catenin signaling pathway, significantly reduced the expression of cardiac-specific genes and partially blocked the role of the miR1-2.

Conclusion: Over-expression of miR1-2 in mouse BMSCs can induce them toward promoted cardiomyocyte differentiation via the activation of the Wnt/ $\beta$-catenin signaling pathway. Compared to 5-aza, miR1-2 can induce differentiation of BMSCs into cardiomyocytes more effectively with a less cytotoxicity.
\end{abstract}

Keywords: miR1-2, Mesenchymal stem cells, Cardiomyocyte differentiation, Wnt/B-catenin signaling pathway

\section{Background}

Cardiovascular diseases are the number one among the disease-caused mortality in the world. The use of stem cells as a potential therapeutic modality has recently been proposed for the treatment of cardiovascular diseases [1, 2]. Embryonic stem cells (ESCs) have been reported to be able to repair the damaged heart muscle and exhibit strong therapeutic potentials [1]. However, the ethical controversies on the origin of ESCs hinder its broad application in human patients. Emerging evidence has shown that bone marrow-derived mesenchymal stem cells (BMSCs) possess

\footnotetext{
* Correspondence: jietian@cqmu.edu.cn

'Department of Cardiology, Heart Centre, The Children's Hospital of Chongqing Medical University, 136 Zhongshan Er Road, Chongqing 400014 Yu Zhong District, China

Full list of author information is available at the end of the article
}

lots of important biological properties such as transdifferentiation, angiogenesis, anti-apoptosis, anti-fibrosis, anti-inflammatory, and immunosuppressing [2, 3]. In fact, BMSCs can turn into a variety of cell types such as cardiomyocytes, osteoblasts, endothelial cells, neurons, and fat cells due to their multi-potent differentiation abilities [2, 3]. But despite the growing attention paid to BMSCs in cardiac repairing,there are still many problems, one of them is the poor differentiation rate of BMSCs into myocardial cells. It has been reported that BMSCs were able to transdifferentiate into cardiomyocyte-like cells after being exposed to 5-azacytine (5-aza) [4], however, 5-aza is an exogenous chemical reagent that would cause cell damage. Therefore, it is very important to find a safe method for inducing differentiation of BMSCs into cardiomyocyte. 
Micro ribonucleic acids (microRNAs, miRs) are endogenous short non-coding RNAs. They have important regulatory functions in a wide range of biological processes, including regulating cell proliferation, apoptosis, and aging [5]. In addition, they are also involved in the development of the heart and the regeneration of the myocardium [6, 7]. Recently, emerging evidence showed that miRNAs were closely associated with stem cells differentiation. It was reported that in cardiac progenitor cells, the over-expression of miR1 and miR499 reduced the rate of cell proliferation and enhanced the differentiation via repressing the HDAC4 or Sox6 [8]. Both miR1 and miR133 levels were increased in the differentiated ESCs, and miR1 was able to promote ESCs differentiation into cardiac lineage, however, miR133 may block the differentiation of myogenic precursors [9]. In mammals, miR1 has two copies, one is for miR1-1, mainly related to skeletal muscle development [10], one is for miR1-2, mainly involved in myocardial development [11]. Therefore, we speculate that miR1-2 plays a major role in differentiation of BMSCs into cardiomyocyte, but few studies have been done on it.

The miR1 has been reported to be able to modulate cardiomyogenesis and maintain the expression of muscle genes via down regulating the Notch or STAT3 signaling pathways [12-14]. While, Huang F et al found there was only downregulation of Hes-1, the downstream target molecule of the Notch pathway, but not regulation upstream molecules of the Notch pathway [14]. Because Notch signaling is initiated via ligand-Notch receptor interactions on neighboring cells [15]. It suggested other signaling pathway may be involved. Wht signaling plays important roles in the differentiation into cardiomyocytes either from tissue regeneration or from pluripotential stem cells,however, its role in differentiation of BMSCs into cardiomyocyte by miR1-2 has not been fully revealed. It is important to determine whether regulation of this pathway after miR1-2 transfection can help to generate sufficient cardiac for therapeutic use in the future.

In this study, we hypothesize that over-expression of miR1-2 in mouse BMSCs can induce their differentiation into cardiomyocytes through $\mathrm{Wnt} / \beta$-catenin signaling pathway. The effect of miR1-2 in the regulation of the differentiation of BMSCs into cardiomyocytes has been determined and also compared with 5-aza, a positive control that can induce the differentiation of BMSCs into cardiomyocytes.

\section{Methods}

\section{Animals}

Male C57BL/6 mice (4 weeks old) were purchased from the Experimental Animal Center of the Chongqing Medical University (certificate number: SYXK (Chongqing) 20070016). All the procedures and experimental protocols were approved by the Experimental Animal Committee of
Chongqing Medical University. All the mice were treated in accordance with the guideline for the Care and Use of Laboratory Animals published by the U.S. National Institute of Health.

\section{BMSCs isolation and culture in vitro}

The isolation and culture of BMSCs from C57BL/6 mice was performed as described previously [16]. In brief, after anesthesia, the femurs of mice were quickly taken out, and bone marrow cells were flushed form the bone marrow cavities into beaker with MesenCult basal medium supplemented with Mesenchymal Stem Cell Stimulatory Supplement (Stem cell Technologies, Vancouver, BC, Canada). Bone marrow cells were harvested and plated into the dishes and then incubated with the Dulbecco's Modified Eagle Medium (DMEM, Hyclone, GE Healthcare Life Sciences,USA) supplemented with $10 \%$ fetal bovine serum (Gibco, Burlington, ON, Canada), penicillin $(100 \mathrm{U} / \mathrm{ml}) /$ streptomycin $(100 \mu \mathrm{g} /$ $\mathrm{ml}$ ) (Sigma-Aldrich, St. Louis, MO) in a humidified atmosphere of $5 \% \mathrm{CO} 2$ at $37{ }^{\circ} \mathrm{C}$. Non-adherent cells were removed carefully after $48 \mathrm{~h}$ and fresh medium was replaced. When primary cultures became almost confluent, the culture was treated with $0.5 \mathrm{ml}$ of $0.25 \%$ trypsin containing $0.02 \%$ ethylene diamine tetraacetic acid (Sigma-Aldrich, St. Louis, MO) for $1 \mathrm{~min}$ at room temperature $\left(25^{\circ} \mathrm{C}\right)$. Cultured BMSCs between passages 3-5 were used for the following experiments.

\section{BMSCs treatment}

The BMSCs were seeded in $60 \mathrm{~mm}$ dishes and treated with $5 \mu$ M 5-aza (Sigma-Aldrich, St. Louis, MO) as previously reported [4] to induce cardiomyocytes differentiation. Then, we transfected $50 \mathrm{nM}$ synthetic miR1-2 mimics (mimics-sense (5'-3'):ACAUACUUCUUUAUG UACCCAUA, mimics-antisense(5'-3'):UGGGUACAUAA AGAAGUAUGUUU, Sangon Biotech, Shanghai, China) by Lipofectamine 2000 (Invitrogen, Carlsbad, CA) into BMSCs to over-expression of miR1-2 in BMSCs according to instructions provided by the manufacturer. In the same manner, miR1-2 mimics negative control (miR1-2mimics NC, NC-sense (5'-3'): UUCUCCGAACGUGUC ACGUTT, NC-antisense( (5'-3'):ACGUGACACGUUCGG AGAATT, Sangon Biotech, Shanghai, China) was transfected as control. BMSCs treated with DMSO (Invitrogen, Carlsbad, CA) was used as the control also. $1 \mu \mathrm{M}$ LGK-974 (Apexbio, America)was added into BMSCs after miR1-2 mimics transfection at $4 \mathrm{~h}$ to inhibit the $\mathrm{WNT} / \beta$-catenin signaling pathways activities as previously reported [17]. After incubation at $24 \mathrm{~h}, 48 \mathrm{~h}$ and $72 \mathrm{~h}$ in a humidified atmosphere of $5 \% \mathrm{CO} 2$ at $37{ }^{\circ} \mathrm{C}$, the cells were collected for the next experiments. 


\section{Apoptosis assay}

The FITC Annexin V Apoptosis Detection kit with PI (Beyotime Biotecnology, Haimen, China) was used to measure the cell apoptosis. After incubation at $48 \mathrm{~h}$ and $72 \mathrm{~h}$ with 5-aza and miR1-2 mimics, the cells were digested with trypsin and washed twice with PBS and resuspended in Annexin $\mathrm{V}$ binding buffer at a concentration of $0.25 \sim 1.0 \times 10^{7}$ cells $/ \mathrm{ml}$. The suspension $(100 \mu \mathrm{l})$ was stained with $5 \mu \mathrm{l}$ of FITC/Annexin V and $10 \mu \mathrm{l}$ of PI, and the cells were gently vortexed and incubated in the dark at $25{ }^{\circ} \mathrm{C}$ temperature for 15 minutes. Subsequently, $400 \mu \mathrm{l}$ Annexin $\mathrm{V}$ binding buffer was added to each tube and then analyzed by flow cytometry (BD FACSCalibur, USA).

\section{Real time polymerase chain reaction}

To examine the expression of miR1-2, GATA binding protein 4(GATA4), homeobox protein 2.5(Nkx2.5), cardiac troponin I (cTnI), $\beta$-catenin, Wnt11, T-cell Factor (TCF), and c-Jun-N-terminal kinase (JNK), we used quantitative real time polymerase chain reaction (qPCR) to measure their mRNA levels. Briefly, total RNAs were isolated and reverse transcribed to cDNA. qPCR was carried out with Tiangen miRNA qPCR kit (FP401, Tiangen, Beijing, China) and Tiangen mRNA qPCR kit (FP302, Tiangen, Beijing, China)with the following parameters: pre-denaturation at $94{ }^{\circ} \mathrm{C}$ for $2 \mathrm{~min}$, followed by 40 cycles of denaturation at $94{ }^{\circ} \mathrm{C}$ for $10 \mathrm{~s}$, annealing at $60{ }^{\circ} \mathrm{C}$ for $34 \mathrm{~s}$. The primers used in the study are listed in Table 1 and the experiments were performed in triplicate. The levels of miR1-2 were normalized against U6 snRNA. The levels of GATA4, Nkx2.5, cTnI, $\beta$-catenin, Wnt11, TCF, and JNK were normalized against GAPDH.

\section{Western blotting}

Proteins were extracted and quantified as previously reported [18]. Each sample containing equal amounts of protein $(30 \mu \mathrm{g})$ was subjected to SDS-PAGE and then was electro-blotted onto PVDF membranes. Immunoblotting and detection of the GATA4(1:1000, Abcam, English), Nkx2.5(1:1000,Abcam, English) and cTnI (1:5000) expression were performed. The immunoreactive bands were revealed with enhanced chemiluminescence (Millipore, Billerica, USA), and analyzed using Quantity One Version 4.62 software (Bio-Rad, Richmond, CA). The $\beta$-actin was used as the internal controls.

\section{Data analysis}

All statistical analyses were calculated using GraphPad Prism (version6.0, Graphpad Software, SanDiego, USA). Data are expressed as mean \pm SEM of three separate experiments. To test the difference between the groups in biochemical measurements for statistical significance, normally distributed data were analyzed by Holm-Sidak
Table 1 Primers used in qPCR assays

\begin{tabular}{|c|c|}
\hline \multicolumn{2}{|c|}{ qPCR primer } \\
\hline \multirow[t]{2}{*}{ U6 } & Forward 5'-CCTGCGCAAGGATGAC-3' \\
\hline & Reverse 5'-GTGCAGGGTCCGAGGT-3' \\
\hline \multirow[t]{2}{*}{ Nkx2.5 } & Forward 5'-ACTTCGTGAACTTTGGCGTC -3' \\
\hline & Reverse 5'-AGGGCATAGTGGGAGCTITC-3' \\
\hline \multirow[t]{2}{*}{ cTnl } & Forward 5'-TTCTGAGGACTCGTTGCCAG -3' \\
\hline & Reverse 5'-ATCCACTTTGTCCACCCGAG-3' \\
\hline \multirow[t]{2}{*}{ GATA4 } & Forward 5'-TCTCACTATGGGCACAGCAG-3' \\
\hline & Reverse 5'-GCGATGTCTGAGTGACAGGA-3' \\
\hline \multirow[t]{2}{*}{ Wnt11 } & Forward 5'-CCAAATCTCTGCCCTCCTCA-3' \\
\hline & Reverse 5'-CCTCACCCTITGACCAACAGA-3' \\
\hline \multirow[t]{2}{*}{$\beta$-catenin } & Forward 5'-GCAGTGAAGAATGCACACGA-3' \\
\hline & Reverse 5'-CAAGCAAAGTCAGCACCACT-3' \\
\hline \multirow[t]{2}{*}{ TCF } & Forward 5'-ATTAGCGAGAGGGTCTGAGC-3' \\
\hline & Reverse 5'-AGTTTTGCACACGGTCAGTC-3' \\
\hline \multirow[t]{2}{*}{ JNK } & Forward 5'-TGGAGTCATAAGAGGGCAGC-3' \\
\hline & Reverse 5'-ACTGCTGTCTGTATCCGAGG-3' \\
\hline \multirow[t]{2}{*}{ GAPDH } & Forward 5'-ACCTGACCTGCCGTCTAGAA-3' \\
\hline & Reverse 5'-TCCACCACCCTGTTGCTGTA-3' \\
\hline
\end{tabular}

tests for multiple group comparisons, and students t-test for two groups comparisons. Data that did not meet the assumptions of analysis were analyzed by the Mann-Whitney $\mathrm{U}$ test. We regarded P values of $<0.05$ as significant.

\section{Results}

Expression levels of miR1-2 in BMSCs treated with 5-aza and miR1-2

We used 5-aza to treat BMSCs, we found the expression of miR1-2 did not change at 24 hours, but significantly increased 1.6 times at $48 \mathrm{~h}$ and 3.0 times at $72 \mathrm{~h}$ comparing to control after 5-aza intervention, which indicates that the level of miR1-2 was increased in 5-aza-induced BMSCs to differentiate into cardiomyocytes (Fig. 1a). Then, we transfected miR1-2 mimics into BMSCs, 24 hours after the treatment, the expression of miR1-2 in BMSCs was significantly increased, although the level of miR1-2 decreased with time, it was still 600-fold higher than that of the control group after transfection of $72 \mathrm{~h}$, (Fig. 1b), indicating that miR1-2 mimics mediated miR12 expression in BMSCs.

Effect of 5-aza and miR-1 mimic on apoptosis in BMSCs We investigated whether 5-aza and miR1-2 mimics treatment could induce apoptosis. Flow cytometry analysis showed that there was no significant difference in the rate of apoptosis in BMSCs after 5-aza and miR1-2 mimics treatment at $48 \mathrm{~h}$ (Fig. 2a). However, the rate of apoptosis was significantly increased after $72 \mathrm{~h}$ treatment 

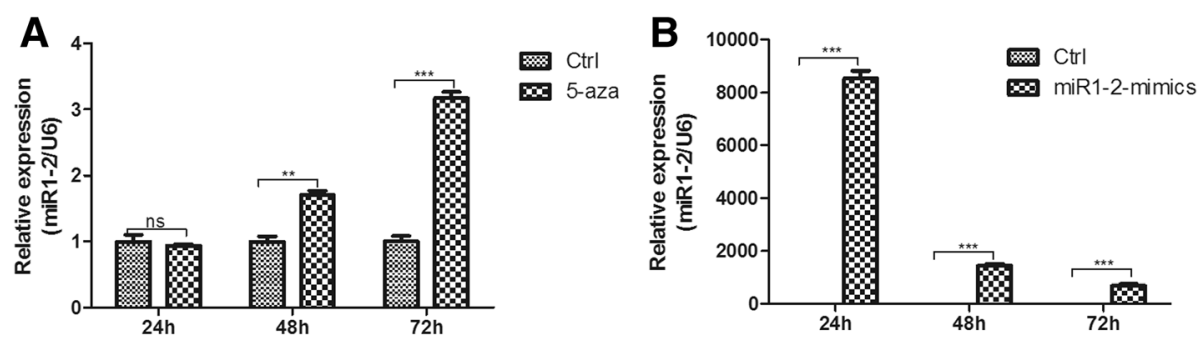

Fig. 1 The expression of miR1-2 in BMSCs. The expression of miR1-2 was significantly increased in BMSCs treated with 5-aza and miR1-2 mimics. a 5-aza treatment. b miR1-2 mimics treatment. ns, $p>0.05,{ }^{* *}, p<0.01,{ }^{* * *}, p<0.001$, compared to controls

with 5 -aza $(16.51 \%$ increase), not in miR1-2mimics group (Fig. 2b), indicating that unlike 5-aza, miR1-2 does not induce cell apoptosis (Fig. 2c)

\section{Over-expression of miR1-2 induces expression of cardiac- specific genes in BMSCs}

We then asked whether the over-expression of miR1-2 in BMSCs could induce the differentiation of these cells into cardiomyocytes. The expression levels of several cardiacspecific genes, such as GATA4, c'TnI and Nkx2.5 were examined using qPCR. Our data indicated that the expressions of GATA4 (Fig. 3a), Nkx2.5 (Fig. 3b) and cTnI (Fig. 3c) were significantly increased after $48 \mathrm{~h}$ treatment with 5-aza, but they decreased with time, the expression of GATA4 even has not significantly increased compared to control group after treatment at $72 \mathrm{~h}$ (Fig. 3a). After transfection of miR1-2 mimics at $48 \mathrm{~h}$, the expression of GATA4 (Fig. 3a), Nkx2.5 (Fig. 3b) and cTnI (Fig. 3c) significantly increased also compared to the control, and they increased with time, after $72 \mathrm{~h}$ of treatment, the expression of these genes in miR1-2 group were significantly higher than those of the 5-aza group and the control group (Fig. 3). These results indicated that over-expression of miR1-2 could induce BMSCs differentiation into cardiac cells by enhancing the expression levels of the cardiac genes.

\section{Over-expression of miR1-2 activates $\mathrm{Wnt} / \boldsymbol{\beta}$-catenin signal- ing pathway}

Previous studies strongly implied that miR-1 promoted cardiac differentiation in human embryonic stem cells,

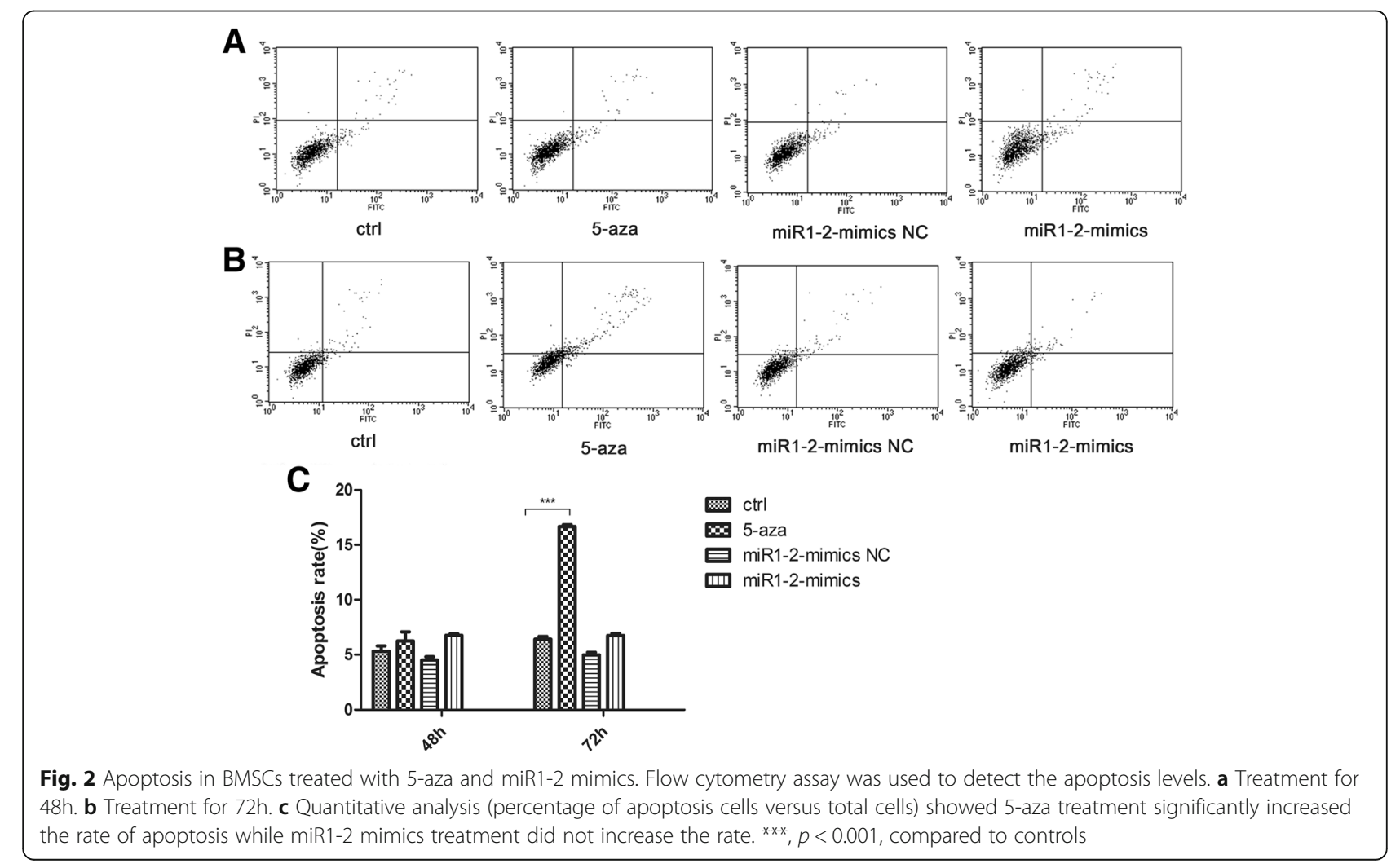



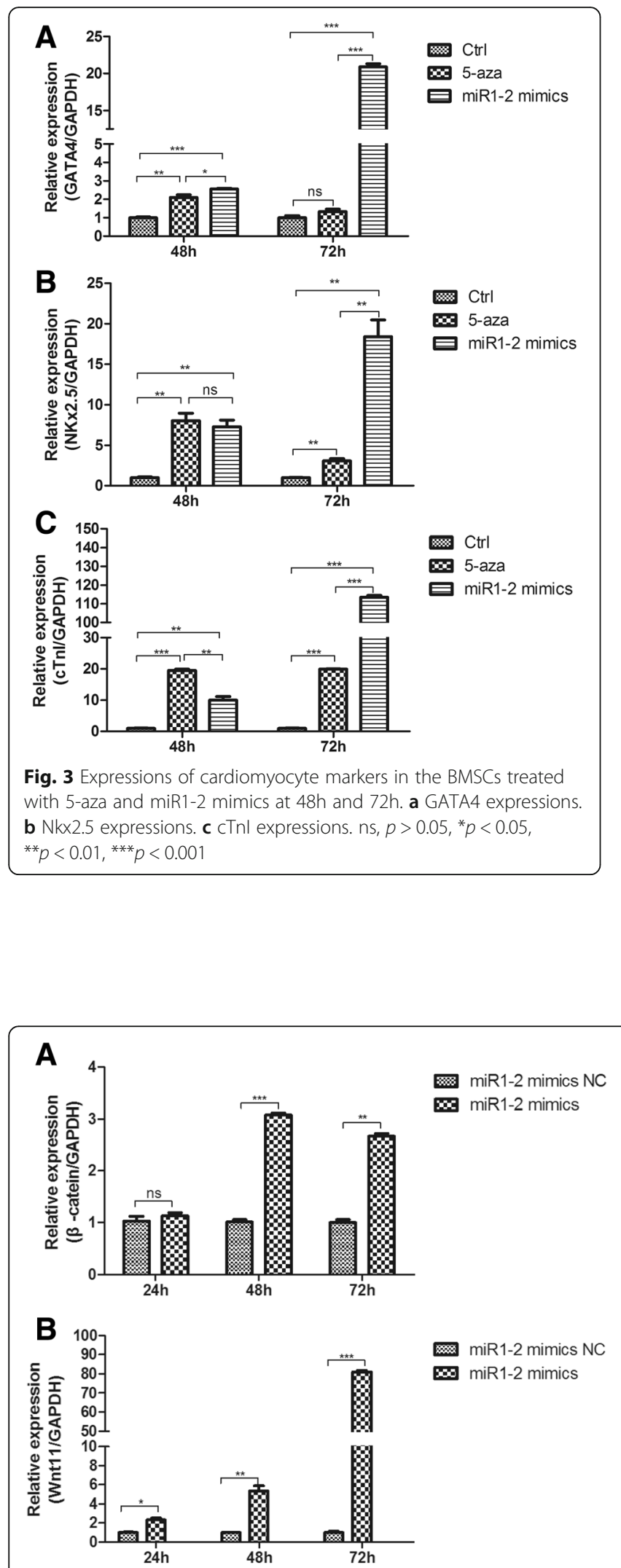

cardiac stem cells, or cardiac progenitor cells via regulating the activity of the Wnt/ $\beta$-catenin signaling pathway. We therefore asked whether the over-expression of miR1-2 induces cardiac differentiation of mouse BMSCs through the $\mathrm{Wnt} / \beta$-catenin signaling pathway. For this purpose, we measured the transcription levels of upstream protein including $\beta$-catenin, wnt11, TCF, and JNK of Wnt/ $\beta$-catenin pathway in BMSCs after miR1-2 mimic treatment. The results showed that the relative expression of Wnt11 (Fig. 4b), TCF (Fig. 4c), and JNK (Fig. 4d) was significantly increased after $24 \mathrm{~h}$ treatment with miR1-2 mimics compared to the miR1-2 mimics NC. Though the expression of $\beta$-catenin was not increased at $24 \mathrm{~h}$, but it significantly increased at $48 \mathrm{~h}$ and 72h (Fig. 4a). These results indicated that Wnt signaling pathway may play important roles in the differentiation of BMSCs into cardiomyocytes by miR1-2 induced.

\section{miR1-2 induces cardiac differentiation through Wnt/ $\beta$ - catenin signaling activation}

We then asked whether the increased level of cardiacspecific genes induced by the over-expression of miR1-2 is through the Wnt signaling. For this purpose, we used LGK-974, an inhibitor of Wnt/ $\beta$-catenin signaling pathway to suppress the activation of $\mathrm{Wnt} / \beta$-catenin signaling pathway. As a result, the relative expression of $\beta$-catenin, JNK, Wnt11 and TCF was significantly increased after miR1-2 mimic treatment, but the levels of $\beta$-catenin together with JNK, Wnt11 and TCF were significantly decreased after adding LGK-974 (Fig. 5). In parallel to the data from qPCR analysis, WB analysis also confirmed that after treated with miR1-2mimics at $72 \mathrm{~h}$,

Fig. 4 Expression levels of $\beta$-catenin, Wnt11, TCF, and JNK in BMSCs. The expression levels of $\beta$-catenin (a) Wnt11 (b) TCF (b) and JNK (d) were significantly increased in the BMSCs after miR1-2 mimics treatment. $\mathrm{ns}, p>0.05,{ }^{*}, p<0.05,{ }^{* *}, p<0.01,{ }^{* * *}, p<0.001$, compared to miR1-2 mimics NC 


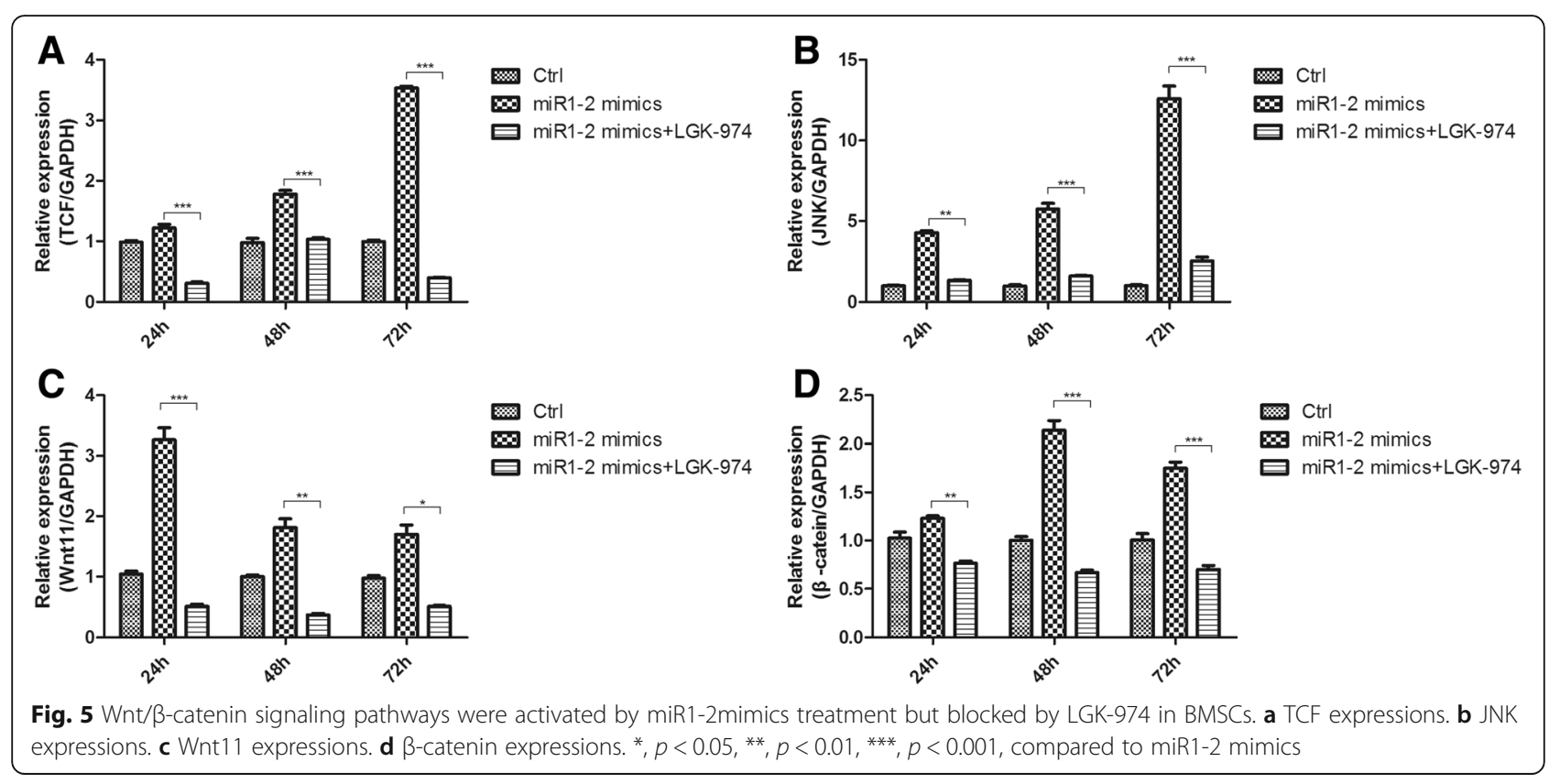

BMSCs increased the expression of the protein of GATA4, NKx 2.5 and cTnI, while treated by Wnt inhibitor, the BMSCs exhibited a distinct reduction of the protein of GATA4, Nkx2.5 and cTnI, compared to the miR1-2 mimics group without Wnt inhibitor treatment (Fig. 6)

\section{Discussions}

It has been documented that BMSCs were capable of turning differentiating into cardiomyocytes, endothelial cells, and vascular smooth muscle cells $[2-4,19]$. In this study, we found that BMSCs treated with miR1-2 mimics displayed the increased expression of cardiac-specific markers such as GATA4 and Nkx2.5. The levels of those cardiac-specific markers were significantly higher than those in 5-aza group. This was consistent with the previous reports that miR-1 induced the expression of several cardiomyocyte markers, including Nkx2.5, GATA4, cTnI, and $\mathrm{Cx} 43$ in $\mathrm{MSCs}^{14}$. It was reported that translocation of Nkx2.5 and GATA4 to the nucleus can drive BMSCs to Cardiac phenotype [19], miR-1 could upregulate Nkx2.5 to promote Cardiac differentiation [20]. We also observed that the apoptosis rate of the miR1-2 mimics group was significantly lower than the 5-aza group. Carley et al also reported that miRNA1 transfected embryonic stem cells could inhibit apoptosis by modulating the PTEN/Akt pathway in the infarcted heart [21].All of these results suggested that miR1-2 could induce the differentiation of BMSCs into cardiomyocytes, and this method may be more effective and less cytotoxic.

Wnt signaling pathway plays an essential role during embryonic and postnatal cardiomyocytes development. Previous studies has showed that $\beta$-catenin in Drosophila could promote the occurrence of the heart [22].Wnt11 could regulate cell adhesion and affect the formation of cardiac linear tube, knockdown wnt11 would lead the defects of ventricular outflow tract, and the reduction of cardiac trabecular in mouse [23]. The Wnt signaling pathway also simultaneously regulates the proliferation and the differentiation of BMSCs [24]. Moreover, miRNA could regulate gene expression and act as important factors in the cardiomyocytes development and the differentiation of stem cell [12-14].Therefore, these observations raised an interesting question whether miRNA could modulate signaling pathway to regulate the differentiation of stem cell. Recently, accumulated evidences indicated that miRNA could regulate various bioprocesses by targeting Wnt signaling pathways. For example, miR218 was reported to be able to promote the differentiation of BMSCs by activating a positive Wnt signaling loop [25]. miR27 inhibits the gene expression of adenomatous polyposis coli leading $\beta$-catenin accumulation and thus Wnt signaling pathways activation to promote osteoblast differentiation[26]. Tcf-1 is a critical target gene of the Wnt/ $\beta$-catenin signaling pathways [27]. Inhibiting low density lipoprotein receptor-related protein 6 by miR30e over expression significantly downregulates $\beta$-catenin/Tcf transcriptional activity and dramatically inhibits osteoblasts differentiation [28]. All these results are accordant with our findings in this study that the over-expression of miR1-2 activated Wnt/ $\beta$-catenin pathway as evidenced by the findings that the expression of $\beta$-catenin, Wnt 11 , TCF, and JNK were significantly increased. After using LGK-974, a highly potent and selective Wnt signaling antagonist [29] to block the Wnt/ $\beta$-catenin signaling, the activity of the Wnt/ $\beta$-catenin signaling pathways and the expression of cardiac-genes both decreased. These data 


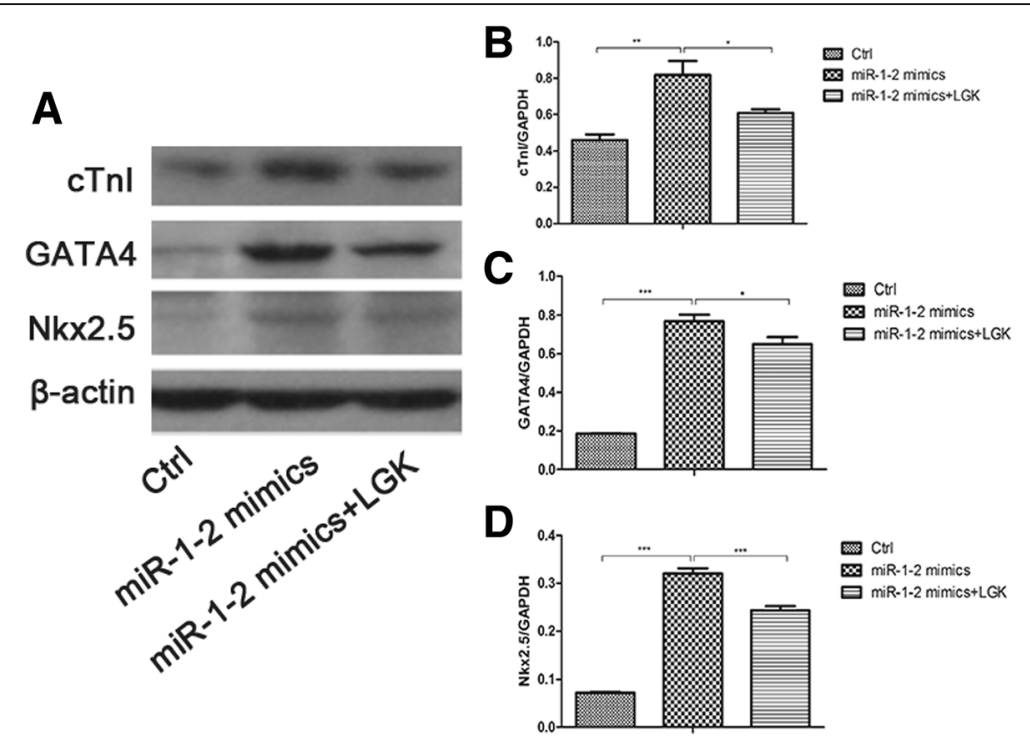

Fig. 6 Expression of cardiac protein in BMSCs after miR1-2mimics treatment. a Western blot band. $\mathbf{b}, \mathbf{c}$, $\mathbf{d}$ WB analysis confirmed that miR12 mimics treatment increased the expression of $\mathrm{cTnl}(\mathrm{B})$, GATA4(C), and Nkx2.5(D) in mouse BMSCs, ${ }^{* *}, p<0.01,{ }^{* * *}, p<0.001$, compared to controls. While using LGK-974(Wnt inhibitor) exhibited a distinct reduction of the protein expression of cTnl(B), GATA4(C) and Nkx 2.5(D). * $p<0.05,{ }^{* * *}, p$ $<0.001$, compared to miR1-2 mimics

indicated miR1-2 might promote the differentiation of BMSCs into cardiomyocytes via activation of the Wnt/ $\mathrm{B}$ catenin signaling pathways. However, we did not find a decrease in expression levels in the molecules of the detected Wnt signaling pathways. It's controversy to the miRNA function post-transcriptionally by interacting directly with 3'-UTRs of mRNAs to repress their expression by translational inhibition, mRNA degradation, or both [30].Maybe, it suggests the existence of undefined miR1-2 target genes, which could inhibit Wnt signaling. It has been reported that miR-335-5p activated Wnt signaling and promoted osteogenic differentiation by downregulating Dickkopf-related protein 1 (DKK1), an inhibitor of Wnt signaling [31]. Yalan Yang et al also found that miR1/206 targeted Secreted frizzled-related protein one (SFRP1), another inhibitor of Wnt signaling to promote skeletal muscle development [32].

Although in our study, BMSCs treated with miR1-2 expressed cardiac-specific genes, these cells still lacked the morphology of cardiomyocytes and did not beat by themselves, which were observed in studies with embryonic stem cells [33]. In addition, the undefined target genes of microRNA1-2 warrant further investigation.

\section{Conclusions}

In summary, our study demonstrated that the overexpression of miR1-2 in BMSCS induced them toward cardiomyocyte differentiation through activating $\mathrm{Wnt} / \beta$-catenin signaling pathways, and miR1-2 can induce BMSCs differentiation into cardiomyocytes more effectively with a less cytotoxicity than that of 5-aza. These findings will greatly improve our understanding of the roles of miRNA in cardiomyocyte differentiation of stem cells and improve the effects of BMSCs-based therapy for damaged myocardial repair and regeneration.

Abbreviations

microRNA: micro ribonucleic acid; BMSCs: Bone marrow-derived mesenchymal stem cells; cTnl: Cardiac troponin I; Nkx2.5: Homeobox protein 2.5; GATA4: GATA binding protein 4; JNK: c-Jun-N-terminal kinase; TCF: T-cell Factor

\section{Acknowledgements}

Not applicable.

\section{Funding}

This study was supported by research grants from Luzhou Municipal Government - Southwest Medical University Joint Fund (Grant Number: 2015 lzcyds05 (9/12)).

\section{Availability of data and materials}

The datasets used and/or analysed during the current study are available from the corresponding author on reasonable request.

\section{Authors' contributions}

XS carried out the GPCR and Western blotting assays and data analyses. BP and $\mathrm{HZ}$ carried out the apoptosis assays. LL, LT and JZ participated in the result discussion and data analyses. XS, BP, JT and XH participated in experimental design and manuscript drafting. All authors read and approved the final manuscript.

\section{Competing interests}

The authors have no conflicts of interest to declare.

\section{Consent for publication}

Not applicable.

\section{Ethics approval and consent to participate}

All experiments were purchased from the Experimental of Chongqing Medical University (certificate number: SYXK (Chongqing) 2007-0016). All the mice were treated in accordance with the guideline for the Care and Use of Laboratory Animals published by the U.S. National Institute of Health. 


\section{Publisher's Note}

Springer Nature remains neutral with regard to jurisdictional claims in published maps and institutional affiliations.

\section{Author details}

'Department of Cardiology, Heart Centre, The Children's Hospital of Chongqing Medical University, 136 Zhongshan Er Road, Chongqing 400014, Yu Zhong District, China. ${ }^{2}$ Department of Pediatrics, the Affiliated Hospital of Southwest Medical University, LuZhou, Sichuan 646000, China. ${ }^{3}$ Department of Biomedical Science, Charlie E. Schmidt College of Medicine, Florida Atlantic University, Boca Raton, FL 33431, USA.

Received: 15 March 2017 Accepted: 3 May 2017

Published online: 10 May 2017

\section{References}

1. Mignone $\mathrm{J}$, Kreutziger $\mathrm{KL}$, Paige $\mathrm{SL}$, et al. Cardiogenesis from human embryonic stem cells. Circ J. 2010;74(12):2517-26.

2. Singh A, Singh A, Sen D. Mesenchymal stem cells in cardiac regeneration: a detailed progress report of the last 6 years (2010-2015). Stem Cell Res Ther. 2016;7(1):82. doi:10.1186/s13287-016-0341-0.

3. Silva GV, Litovsky S, Assad JA, Sousa AL, Martin BJ, Vela D, et al. Mesenchymal stem cells differentiate into an endothelial phenotype, enhance vascular density, and improve heart function in a canine chronic ischemia model. Circulation. 2005;111:150-6.

4. Makino S, Fukuda K, Miyoshi S, Kodama H, Fan J, et al. Cardiomyocytes can be generated from marrow stromal cells in vitro. J Clin Invest. 1999;103(5):697-705.

5. Francis N, Moore M, Asan SG, Rutter GA, Burns C. Changes in microRNA expression during differentiation of embryonic and induced pluripotent stem cells to definitive endoderm. Gene Expr Patterns. 2015;19(1-2):70-82. doi:10.1016/j.gep.2015.08.001

6. Chen JF, Murchison EP, Tang R, Callis TE, Tatsuguchi M, Deng Z, et al. Targeted deletion of Dicer in the heart leads to dilated cardiomyopathy and heart failure. Proc Natl Acad Sci U S A. 2008;105(6):2111-6.

7. Porrello ER, Johnson BA, Aurora AB, Simpson E, Nam YJ, Matkovich SJ, et al. miR-15 family regu1ates postnata1 mitotic arrest of cardiomyocytes. Circ Res. 2011;109(6):670-9.

8. Sluijter JP, van Mil A, van Vliet P, Metz CH, Liu J, Doevendans PA, et al. MicroRNA-1and-499 regulate differentiation and proliferation in humanderived cardiomyocyte progenitor cells. Arterioscler Thromb Vasc Biol. 2010;30:859-68.

9. Takaya T, Ono K, Kawamura T, Takanabe R, Kaichi S, Morimoto T, et al. MicroRNA-1 and MicroRNA-133 in spontaneous myocardial differentiation of mouse embryonic stem cells. Circ J. 2009;73(8):1492-7.

10. Tyler J, Thomas C, John J. The role of microRNAs in skeletal muscle health and disease. FrontBiosci. 2015;20:37-77.

11. Zhao Y, Ransom JF, Li A, Vedantham V, von Drehle M, Muth AN, et al. Dysregulation of cardiogenesis, cardiac conduction, and cell cycle in mice lacking miRNA-1-2. Cell. 2007;129(2):303-17.

12. Kwon C, Han Z, Olson EN, Srivastava D. MicroRNA1 influences cardiac differentiation in Drosophila and regulates Notch signaling. Proc Natl Acad Sci U S A. 2005;102:18986-91.

13. Car B, Li J, Wang J, Luo X, Ai J, Liu Y, et al. microRNA-124 regulates cardiomyocyte differentiation of bone marrow-derived mesenchymal stem cells via targeting STAT3 signaling. Stem Cells. 2012;30:1746-55.

14. Huang F, Tang L, Fang ZF, Hu XQ, Pan JY, et al. Corrigendum to "miR-1Mediated Induction of Cardiogenesis in Mesenchymal Stem Cells via Downregulation of Hes-1". Biomed Res Int. 2016;2016:8510747.

15. Luxan G, D'Amato G, MacGrogan D, de la Pompa JL. Endocardial notch signaling in cardiac development and disease. Circ Res. 2016;118(1):e1-e18. doi:10.1161/CIRCRESAHA.115.305350.

16. Soleimani M, Nadri S. A protocol for isolation and culture of mesenchymal stem cells from mouse bone marrow. Nat Protoc. 2009;4:102-6.

17. Jiang X, Hao HX, Growney JD, Woolfenden S, Bottiglio C, Ng N, et al. Inactivating mutations of RNF43 confer Wht dependency in pancreatic ductal adenocarcinoma. Proc Natl Acad Sci U S A. 2013;110(31):12649-54.

18. Pan B, Zhu J, Lv T, Sun H, Huang $X$, Tian J. Alcohol consumption during gestation causes histone 3 lysine 9 hyperacetylation and an alternation of expression of heart development-related genes in mice. Alcohol Clin Exp Res. 2014;38(9):2396-402
19. Arimna A, Ganda C, Bartual M, Garcia-Verdugo JM, Lledo E, Mirabet V, et al. Cardiac differentiation is driven by NKx2.5 and GATA4 nuclear translocation in tissue-speciffec mesenchymal stem cells. Stem Cells Dev. 2009;18:907-18.

20. Ivey KN, Muth A, Arnold J, King FW, Yeh RF, Fish JE, et al. microRNA regulation of cell lineages in mouse and human embryonic stem cells. Cell Stem Cell. 2008:2:219-29.

21. Carley G, Dinender KS. MicroRNA-1 transfected embryonic stem cells enhance cardiac myocyte differentiation and inhibit apoptosis by modulating the PTEN/Akt pathway in the infarcted heart. Am J Physiol Heart Circ Physiol. 2011;301(5):H2038-49.

22. Kim YS, Kim MJ, Koo TH, Kim JD, Koun S, Ham HJ, et al. Histone deacetylase is required for the activation of Wnt/ $\beta$-catenin signaling crucial for heart valve formation in zebrafish embryos. Biochem Biophys Res Commun. 2012;423(1):140-6.

23. Nagy II, Railo A, Rapila R, Hast T, Sormunen R, Tavi P, et al. Wnt-11 signalling controls ventricular myocardium development by patterning $\mathrm{N}$-cadherin and beta-catenin expression. Cardiovasc Res. 2010;85(1):100-9.

24. Gwak J, Hwang SG, Park HS, Choi SR, Park SH, Kim H, et al. Small moleculebased disruption of the Axin/beta-catenin protein complex regulates mesenchymal stem cell differentiation. Cell Res. 2012;22:237-47.

25. Hassan MQ, Maeda Y, Taipaleenmaki H, Zhang W, Jafferji M, Gordon JA, et al. miR-218 directs a Wnt signaling circuit to promote differentiation of osteoblasts and osteomimicry of metastatic cancer cells. J Biol Chem. 2012:287:42084-92

26. Wag T, Xu Z. miR-27 promotes osteoblasts differentiation by modulating Wnt signaling. Biochem Biophys Res Commun. 2010:402:186-9.

27. Travis A, Amsterdam A, Belanger C, Grosschedl R. LEF-1, a gene encoding a Iymphoid-specific protein with an HMG domain, regulates T-cell receptor alpha enhance function. Genes Dev. 1991;5:880-94.

28. Wang J, Guan X, Guo F, Zhou J, Chang A, Sun B, et al. miR-30e reciprocally regulates the differentiation of adipocytes and osteoblasts by directly targeting low-density lipoprotein receptor-related protein 6. Cell Death Dis. 2013:4:e845. doi:10.1038/cddis.2013.356.

29. Liu J, Pan S, Hsieh MH, Ng N, Sun F, Wang T, et al. Targeting Wnt-driven cancer through the inhibition of Porcupine by LGK974. Proc Natl Acad Sci U S A. 2013;110:20224-9.

30. Bartel DP. MicroRNAs: genomics, biogenesis, mechanism, and function. Cell. 2004;116(2):281-97.

31. Zhang J, Tu Q, Bonewald LF, He X, Stein G, Lian J, et al. Effects of miR-335$5 p$ in modulating osteogenic differentiation by specifically downregulating Wnt antagonist DKK1. J Bone Miner Res. 2011;26(8):1953-63. doi:10.1002/ jbmr.377.

32. Yang Y, Sun W, Wang R, Lei C, Zhou R, Tang Z, Li K. Wnt antagonist, secreted frizzled-related protein 1, is involved in prenatal skeletal muscle development and is a target of miRNA-1/206 in pigs. BMC Mol Biol. 2015;16:4.

33. Fu JD, Rushing SN, Lieu DK, Chan CW, Kong CW, Geng L, et al. Distinct roles of microRNA-1and -499 in ventricular specification and functional maturation of human embryonicstem cells-derivedcardiomyocytes. PLoS One. 2011;6(11):e27417. doi:10.1371/journal.pone.0027417.

\section{Submit your next manuscript to BioMed Central and we will help you at every step:}

- We accept pre-submission inquiries

- Our selector tool helps you to find the most relevant journal

- We provide round the clock customer support

- Convenient online submission

- Thorough peer review

- Inclusion in PubMed and all major indexing services

- Maximum visibility for your research

Submit your manuscript at www.biomedcentral.com/submit
) Biomed Central 